\title{
Dynamic effects of chronic unpredictable mild stress on the hippocampal transcriptome in rats
}

\author{
FENG LI, YING WANG, XUE WANG, YUN ZHAO, FANG XIE and LING-JIA QIAN \\ Department of Military Cognitive and Stress Medicine, Beijing Institute of Basic Medical Sciences, \\ Academy of Military Medical Sciences, Beijing 100039, P.R. China
}

Received November 15, 2021; Accepted January 17, 2022

DOI: $10.3892 / \mathrm{mmr} .2022 .12626$

\begin{abstract}
Stress causes extensive changes in hippocampal genomic expression, leading to changes in hippocampal structure and function. The dynamic changes in hippocampal gene expression caused by stress of different durations are still unknown. mRNA sequencing was used to analyze the hippocampal transcriptome of rats subjected to chronic unpredictable mild stress (CUMS) of different durations. Compared with the control, 501, 442 and 235 differentially expressed genes (DEGs) were detected in the hippocampus of rats subjected to CUMS for 3 days and 2 and 6 weeks, respectively. Gene Ontology (GO) analysis was used to determine the potential mechanism underlying the dynamic harmful effects of stress on the hippocampus; Certain GO terms of the down-regulated DEGs in CUMS (3 days) rats were also found in the up-regulated DEGs in CUMS (6 weeks) rats. These results showed opposing regulation patterns of DEGs between CUMS at 3 days and 6 weeks, which suggested a functional change from adaptation to damage in during the early and late stages of chronic stress. GO analysis for upregulated genes in rats subjected to CUMS for 3 days and 2 weeks suggested significant changes in 'extracellular matrix' and 'wound healing'. Upregulated genes in rats subjected to CUMS for 2 weeks were involved in changes associated with visual function. GO analysis of DEGs in rats subjected to CUMS for 6 weeks revealed increased expression of genes associated with 'apoptotic process' and 'aging' and decreased expression of those associated with inhibition of cell proliferation and cell structure. These results suggest that the early and middle stages of chronic stress primarily promote adaptive regulation and damage repair in
\end{abstract}

Correspondence to: Dr Fang Xie or Professor Ling-Jia Qian, Department of Military Cognitive and Stress Medicine, Beijing Institute of Basic Medical Sciences, Academy of Military Medical Sciences, 27 Taiping Road, Haidian, Beijing 100039, P.R. China

E-mail:vancoxie@sina.com

E-mail: newjia@vip.sina.com

Key words: hippocampus, chronic unpredictable mild stress, mRNA sequencing, genetic association study the organism, while the late stage of chronic stress leads to damage in the hippocampus.

\section{Introduction}

Chronic stress is a non-specific complex psychosomatic process that is a key risk factor for depression (1), anxiety (2), drug abuse (3), cardiovascular disease (4), ulcers (5) and cancer (6). Therefore, investigating the positive or negative responses to stressors is important to reveal the pathogenesis of stress-associated disease. The body's response to stressors depends on the nature, intensity and duration of the stressor, forming an 'inverted U'-shaped continuous quantitative effect curve (7). In long-term stress, early stress causes the body to produce compensatory responses, such as increased alertness, sensitivity; as duration of stress increases, the body systems act in concert to maintain homeostasis, thus actively adapting to the stressor. The cumulative effect of further stress leads to a disturbance of homeostasis in the body, resulting in pathophysiological damage (1).

The hippocampus is responsible for learning, memory, cognition and emotion; it is also one of the key brain areas that mediate stress reactions (8). Prolonged stress results in decreased hippocampal nerve cell plasticity, disequilibrium between hippocampal apoptosis and regeneration, leading to nerve cell atrophy and loss and causing structural and functional damage in the hippocampus $(9,10)$. Studies have investigated the effects of different stress patterns on gene expression in the hippocampus $(11,12)$, but, to the best of our knowledge, there are no reports on dynamic changes in gene expression in the hippocampus during chronic stress.

To determine the effects of stress on hippocampal structure, the present study investigated transcriptomic changes in the rat hippocampus during chronic unpredictable mild stress (CUMS) using mRNA sequencing (seq) technology. The CUMS rodent model uses both physiologically and psychologically stressful stimulations to mimic adverse stress from negative life events and induces decreased locomotion and anhedonia, similar to symptoms of depression in humans, which makes it a widely used animal model to study the molecular basis of stress in the brain (13). The present study investigated gene expression in the hippocampus of rats in the early, middle and late stages of CUMS to clarify continuous gene expression changes in the hippocampus 
in CUMS and reveal the pathophysiological mechanism of stress injury.

\section{Materials and methods}

Animals. A total of 36 male Sprague Dawley (SD) rats (age, 2 months; weight, $180-220 \mathrm{~g}$ ) were obtained from SBF (Beijing) Biotechnology Co., Ltd. All animals were housed in groups of three or four in plastic cages and maintained under standard laboratory conditions $\left(12 / 12\right.$-h light/dark cycle, $22 \pm 2^{\circ} \mathrm{C}$, relative humidity $40-70 \%$, ad libitum access to food and water).

CUMS procedure. In brief, SD male rats were randomly divided into four groups ( $\mathrm{n}=9$ per group) as follows: i) Control and CUMS for ii) 3 days and iii) 2and iv) 6 weeks (Fig. 1). The CUMS model was established as previously reported (14): i) Overnight light exposure, ii) darkness for $12 \mathrm{~h}$, iii) wet bedding for $12 \mathrm{~h}$, iv) removal of bedding for $12 \mathrm{~h}, \mathrm{v}$ ) deprivation of food for $12 \mathrm{~h}, \mathrm{vi}$ ) deprivation of water for $12 \mathrm{~h}$, vii) soaking for $3 \mathrm{~min}$ in cold water $\left(4^{\circ} \mathrm{C}\right)$, viii) physical restraint for $4 \mathrm{~h}$, ix) cage vibration $(70 \mathrm{rpm})$ for $2 \mathrm{~h}$ and $\mathrm{x}$ ) electrical shock to the feet for $5 \mathrm{~min}(0.2 \mathrm{~mA})$. Stressors were randomly scheduled to produce an unexpected mild stress effect. A combination of two different stressors was applied separately each day and the same stressor was not repeated within 3 days. Control animals were left uninterrupted except for regular cage cleaning. The effectiveness of the applied stress procedure for induction of the stress response was confirmed by cognitive behavior tests.

Open field test. The test was performed in a black wooden box measuring $100 \times 100 \times 45 \mathrm{~cm}$. The floor was divided into 25 equal squares $(20 \times 20 \mathrm{~cm})$. At the beginning of the experiment, rats were placed individually in the center of the open field and ambulation (number of squares crossed) and head-rise frequency were observed for $5 \mathrm{~min}$ using an ANY-maze behavior analysis system (Stoelting Co.). To ensure that the test results were free from any previous residual effect, the inside of the box was cleaned before each test. An open field test score was calculated as the sum of ambulation and immobility frequency.

Novel object recognition test. The experimental device was a black wooden box $(60 \times 60 \times 45 \mathrm{~cm})$. The test comprised three steps: Adaptation, training and testing. On the day before the experiment, rats were allowed to move freely in the experimental setup (without objects) for $10 \mathrm{~min}$. During the training trial, two identical objects were placed on the left and right ends of a sidewall, and the rats were placed in the field with their back facing the two objects. The animal was placed in the center of the open field and allowed to explore freely for $10 \mathrm{~min}$. During the test, one of the two objects was replaced with another object of different color and shape and the rat was returned to the same box for $10 \mathrm{~min}$. The ANY-maze behavior analysis system recorded the time rats spent exploring the new and old object, denoted as $\mathrm{T}_{\text {new }}$ and $\mathrm{T}_{\text {old }}$, respectively. The cognitive index (CI) was expressed as follows: $\mathrm{CI}=\left(\mathrm{T}_{\text {new }}-\mathrm{T}_{\text {old }}\right) /\left(\mathrm{T}_{\text {new }}+\mathrm{T}_{\text {old }}\right)$.

Morris water maze. The experimental device was a black circular plastic tank (diameter, $160 \mathrm{~cm}$; depth, $75 \mathrm{~cm}$ ) containing water at $22 \pm 2^{\circ} \mathrm{C}$. The curtains around the tank were labeled with stickers of different shapes and colors serving as spatial reference cues. Rats were first required to complete 5 half-days of training at a frequency of once a day for $3 \mathrm{~min}$ each. During training, rats were placed into the maze in one of four quadrants facing the curtain and were trained to find a hidden platform submerged $2 \mathrm{~cm}$ beneath the surface of the water. If rats could not find the platform within $3 \mathrm{~min}$, they were guided to the platform and kept there for $10 \mathrm{sec}$. During the test phase, the platform was removed and rats were placed into the water on the opposite quadrant. Each rat was allowed to swim in the water for $3 \mathrm{~min}$, during which, the first time the rats found the original platform location and the number of platform passes within 3 min were recorded.

Sample preparation. Following behavioral observation, rats were decapitated following anesthesia with sodium pentobarbital $(1 \%, 50 \mathrm{mg} / \mathrm{kg}$, intraperitoneal). The whole brain was removed and washed with cold $0.9 \%$ saline. The hippocampus was dissected on an ice plate on a super-clean bench. The dissected hippocampi were placed into freezing EP tubes, frozen in liquid nitrogen and transferred into a $-80^{\circ} \mathrm{C}$ low-temperature refrigerator for storage and use. RNA was extracted from hippocampal tissue using TRI reagent (Sigma-Aldrich; Merck $\mathrm{KGaA}$ ) according to the manufacturer's protocol. Three hippocampal tissue samples were pooled as a single sample for the total transcript array analysis to increase the quantity of extracted RNA. RNA purity and quantification were evaluated using the NanoDrop 2000 spectrophotometer (Thermo Fisher Scientific, Inc.). RNA integrity was assessed using the Agilent 2100 Bioanalyzer (Agilent Technologies, Inc.). All samples selected for further analysis were of high quality (RNA integrity number $>9,260 / 280-2.1$ ). Then the libraries were constructed using VAHTS Universal V6 RNA-seq Library Prep Kit for Illumina (catalog no. NR604-02; Nanjing Vazyme Biotech Co.) according to the manufacturer's instructions. The concentration of the library used for sequencing was $\geq 3.75 \mathrm{fmol} / \mu \mathrm{l}$ and was measured using a Qubit ssDNA Assay Kit (catalog no. Q10212) and Qubit Fluorometer (catalog no. Q33238) (both; Invitrogen; Thermo Fisher Scientific, Inc.). Transcriptome sequencing and analysis were performed by Shanghai Oe Biotech Co., Ltd.

RNA-seq and differentially expressed gene (DEG) analysis. The libraries were sequenced on MGI DNBSEQ-T7 platform using DNBSEQ-T7RS high-throughput sequencing kit (1000016102, MGI), and 150 bp paired-end reads were generated. A total of $\sim 49.5$ million raw reads were generated for each sample. Raw data (raw reads) of FASTQ format were processed using Trimmomatic (version 0.36) (15) and low-quality reads (adaptor and data of Q30 <85\%) were removed to obtain the clean reads. Then $\sim 49$ million clean reads were retained for each sample for subsequent analysis.

The clean reads were mapped to the rat genome (mRatBN7.2) using Hierarchical Indexing for Spliced Alignment of Transcripts 2 (version 2.2.1.0) (16). Fragments per kilobase of transcript per million mapped reads (FPKM) of each gene was calculated using Cufflinks (version 2.2.1) (17) and the read count of each gene was obtained by HTSeqcount (version 0.9.1) (18). Differential expression analysis was performed using DESeq (2012) R package (19). $\mathrm{P}<0.05$ and 


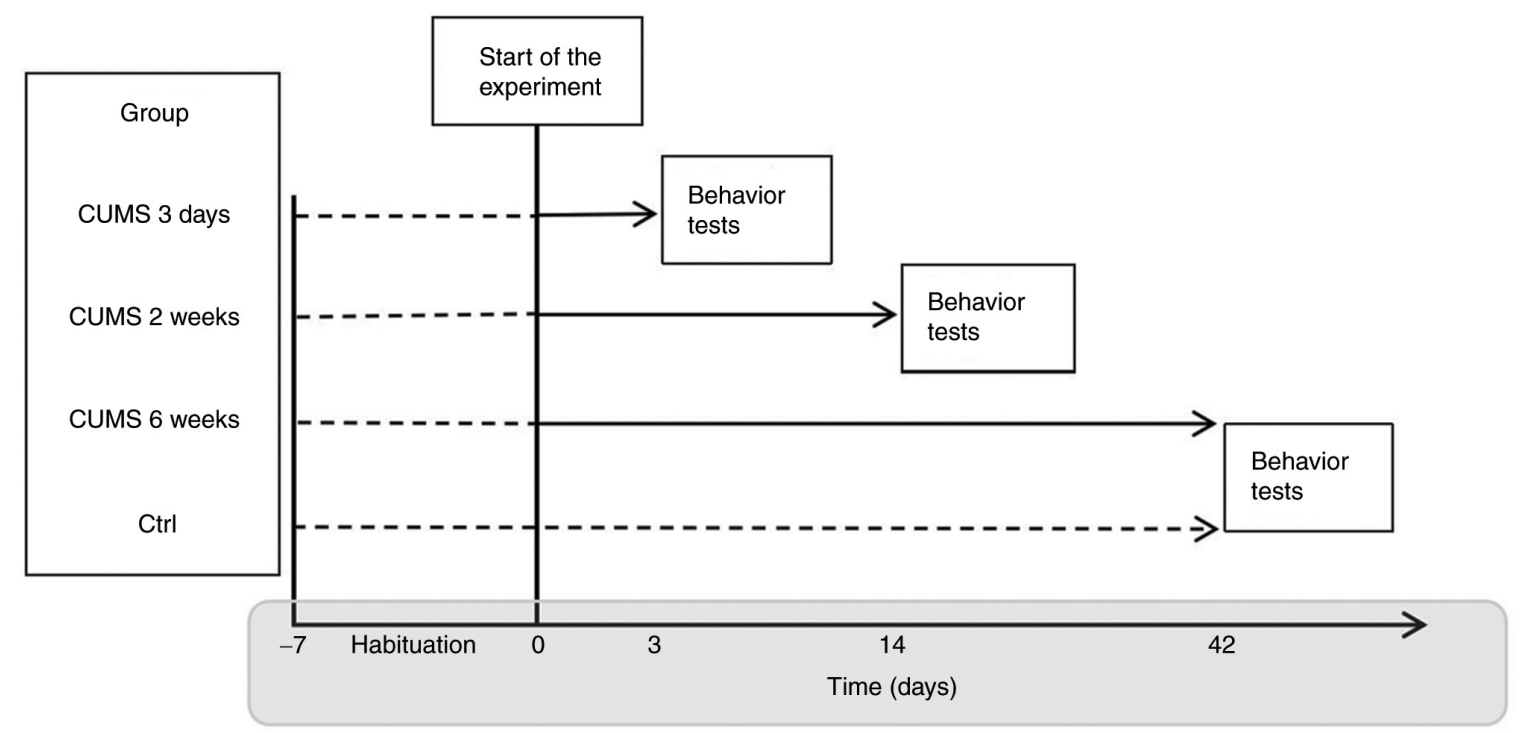

Figure 1. Experimental design. Before the start of the experiment, animals were habituated in the new environment for 7 days. Solid lines mark the days when the animals were stressed. Behavior tests were performed the day after the last stress episode. CUMS, chronic unpredictable mild stress; ctrl, control.

fold-change $>2$ or $<0.5$ was set as the threshold for significantly differential expression.

To identify the functional classes associated with DEGs, the online analysis tool Database for Annotation, Visualization, and Integrated Discovery 6.8 (david.ncifcrf.gov/summary.jsp) and Gene Ontology (GO) database were used to classify the biological process, molecular function, and cellular components of DEGs. GO annotations were obtained from GO database version 2.2 (20). Hypergeometric distributions were used to detect over- or under-represented biological process terms in the studied set compared with the population set. Terms with $\mathrm{P}<0.05$ were considered to be significant.

Confirmation of gene expression levels using reverse transcription-quantitative $(R T-q) P C R$. All selected hippocampal RNA specimens were chosen from the animals used in the mRNA-seq study and were analyzed individually. Meeting three strict conditions $\left[\mathrm{P}<0.05, \log _{2}\right.$ (fold-change) $>1$ and $2<$ average FPKM<50], seven genes were selected to verify the accuracy and reliability of mRNA-seq data. These genes were tested using SYBR-Green-based RT-qPCR in 96-well plates on a LightCycler 96 thermocycler (Roche Diagnostics $\mathrm{GmbH}$ ). Primers (Table I) were designed using Primer-BLAST tool (National Center for Biotechnology Information) with the Rattus norvegicus RefSeq database (reference genome mRatBN7.2). The primers produced amplicons spanning exon-exon junctions and including all known alternatively spliced mRNA variants. A total of 1,000 ng total RNA from each sample was reverse-transcribed to cDNA according to the manufacturer's instructions (ABScript II RT Mix for qPCR, ABclonal Biotech Co., Ltd.). RT-qPCR was run using TB Green Premix Ex Taq II (Takara Bio, Inc.) according to the manufacturer's instructions. The volume of the PCR reaction system, cDNA sample, forward and reverse prime, and TB Green II were 10.0, 1.0, 0.2, 0.2 and $5.0 \mu 1$, respectively. The thermocycling conditions were as follows: Initial denaturation for $2 \mathrm{~min}$ at $95^{\circ} \mathrm{C}$ followed by 50 cycles of $95^{\circ} \mathrm{C}$ for $10 \mathrm{sec}, 60^{\circ} \mathrm{C}$ for $10 \mathrm{sec}$ and $72^{\circ} \mathrm{C}$ for $15 \mathrm{sec}$. All experiments were performed in triplicate. Raw cycle threshold values were calculated on LightCycler. Samples were analyzed by the $\Delta \Delta \mathrm{Cq}$ method. $\Delta \mathrm{Cq}$ values represent normalized target genes levels with respect to the internal control. Normalization was based on a single reference gene ( $\beta$-actin). $\Delta \Delta \mathrm{Cq}$ values were calculated as the $\Delta \mathrm{Cq}$ of each test sample (CUMS) minus the mean $\Delta \mathrm{Cq}$ of the calibrator samples (ctrl) for each target gene. The fold change was calculated using the equation $2^{-\Delta \Delta \mathrm{Cq}}(21)$.

Statistical analysis. The results of behavioral tests were compared using Shapiro-Wilk test. The expression of each mRNA in the hippocampus of CUMS and control groups was compared using Mann-Whitney U test as data did not show a normal distribution. Nine independent experiments were carried out in each group in behavioral tests, while three independent experiments were conducted in each group in RT-qPCR experiments. All data are presented as the mean \pm standard deviation. GraphPad Prism 8 software (ver. 8.4.2; GraphPad Software, Inc.) was used for statistical analysis and Spearman's correlation was used for the correlation analysis. $\mathrm{P}<0.05$ was considered to indicate a statistically significant difference.

\section{Results}

Chronic stress induced time-dependent cognitive decline in rats. Three different behavioral tests were used to evaluate cognitive function in rats. The behavioral results showed that the open field score, object recognition CI and number of platform passes decreased with longer stress time, while the escape latency in the Morris water maze test increased gradually. The difference $(\mathrm{P}<0.05)$ first occurred in the open-field test at 2 weeks of CUMS. All behavioral performance associated with cognitive function decreased significantly $(\mathrm{P}<0.01)$ by week 6 of CUMS, suggesting that chronic stress induced cognitive dysfunction in rats (Fig. S1).

Number of DEGs decreases with the increase in stress time. Compared with the control, 501 DEGs were identified in 
Table I. Primer sequences for reverse transcription-quantitative PCR.

\begin{tabular}{lllrr}
\hline Gene & \multicolumn{1}{c}{ Forward sequence (5'-3') } & \multicolumn{1}{c}{ Reverse sequence (5'-3') } & $\begin{array}{c}\text { Annealing } \\
\text { temperature, }{ }^{\circ} \mathrm{C}\end{array}$ & $\begin{array}{r}\text { Product } \\
\text { size, bp }\end{array}$ \\
\hline Slc6a20 & CTA CAT CCT CAC GGG AAC GC & GTG GCT GAC TTC GGT CTT TG & 60 & 231 \\
Bmp7 & CAT GGA CCC CAG AAC AAG CA & CTT TGG AGT CTT GGA GCG GT & 60 & 123 \\
Ptgds & GTG CAG CCC AAC TTT CAA CA & CCG GAA CCA GCT TGA ATT GG & 60 & 81 \\
Hbb-b1 & TTG GCA GCC TCA GTG AAC TCC & GAC AGA AGC TCT CTT GGG AAC A & 60 & 248 \\
Pdk4 & AAT GTG GTC CCT ACG ATG GC & ATC GCA GTT GGG GTC GAT AC & 60 & 213 \\
Sccpdh & CGA AAC CAG ATG AAC GGC AC & TGC CCT TAT CGC CAA AAC CA & 60 & 127 \\
Npas4 & GTG GCA GCA CTA CCT GGA TT & GTT TGT TGC CTG CAC TCT GG & 60 & 265 \\
Actb & CTT CCT GGG TAT GGA ATC CT & TCT TTA CGG ATG TCA ACG TC & 60 & 80 \\
\hline
\end{tabular}

Slc6a20, solute carrier family 6 member 20; Bmp7, bone morphogenetic protein 7; Ptgds, prostaglandin D2 synthase; Hbb-b1, hemoglobin, $\beta$ adult major chain; Sccpdh, saccharopine dehydrogenase (putative); Npas4, neuronal PAS domain protein 4, Actb, $\beta$-actin.
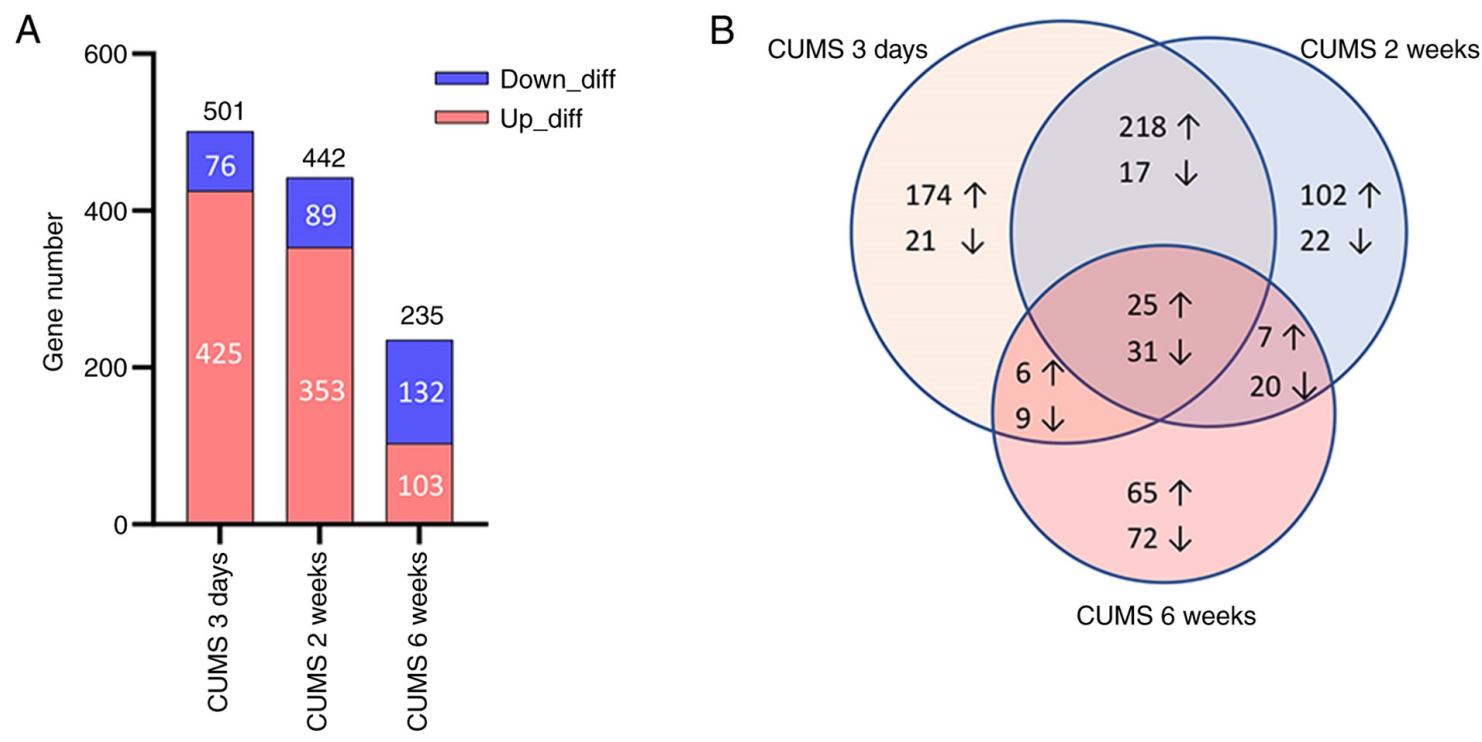

Figure 2. Summary of mRNA sequencing data. (A) Total number of significantly differentially expressed genes and the percentage of up- and downregulated genes. (B) Venn diagram showing gene expression in different groups. CUMS, chronic unpredictable mild stress; ctrl, control.

CUMS (3 days) rats, of which 425 were up- and 76 were downregulated. A total of 442 DEGs in the CUMS (2 weeks) group were identified, of which 353 were up- and 89 were downregulated. A total of 235 DEGs in the CUMS (6 weeks) group were identified, of which 103 were up- and 132 were downregulated (Fig. 2; Table SI). As the duration of stress increased, the total number of DEGs decreased; this was accompanied by a decrease in up- and an increase in downregulated genes.

GO function analysis of DEGs suggests a functional change from adaptation to damage in the early and late stages of chronic stress. DEGs were divided into up- and downregulated groups for GO enrichment analysis. GO terms were divided into three categories: Biological processes, cellular components and molecular functions. The top 10 terms with the most significant P-values $(<0.05)$ were selected from each category. Significant biological processes of upregulated DEGs of the hippocampus in CUMS (3 days) rats included 'collagen fibril organization', 'oxygen transport', 'wound healing', 'cell-matrix adhesion', 'ammonium transmembrane transport', 'cerebrospinal fluid secretion' and 'blood vessel development' (Fig. 3A), while those of downregulated DEGs involved 'positive regulation of neuron projection development', 'inflammatory response', 'multicellular organism development', 'regulation of apoptotic process', 'protein phosphorylation' and 'oxidation-reduction process' (Fig. 3B). Significant biological processes of upregulated DEGs in CUMS (2 weeks) rats included 'visual perception', 'phototransduction', 'retina development in camera-type eye', 'oxygen transport', 'wound healing', 'regulation of cilium beat frequency', 'cerebrospinal fluid secretion', 'collagen fibril organization', 'detection of light stimuli involved in visual perception' and 'receptor-mediated endocytosis' (Fig. 3C), while those of the downregulated DEGs in this group involved 'protein phosphorylation', 'positive regulation of MAPK cascade', 'inflammatory response', 'apoptotic process' and 'intracellular protein transport' (Fig. 3D). Certain biological processes were found after both 3 days 
A

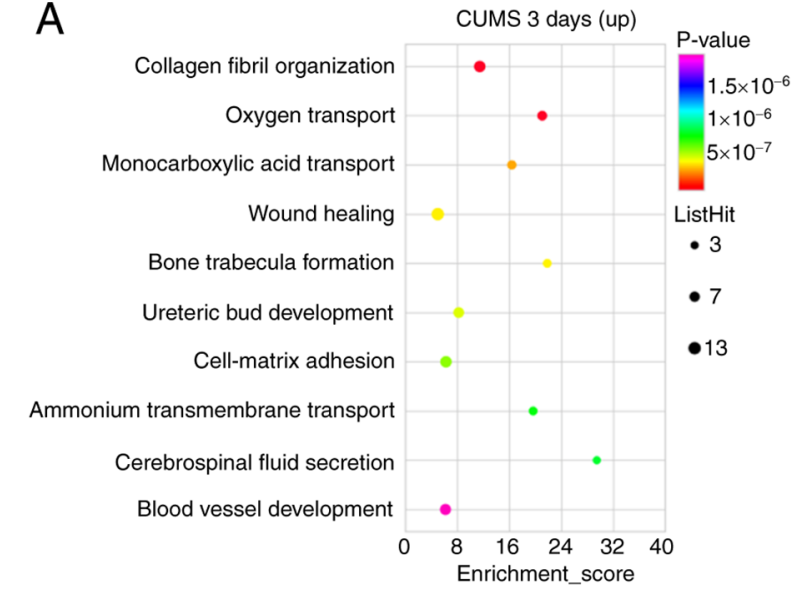

C

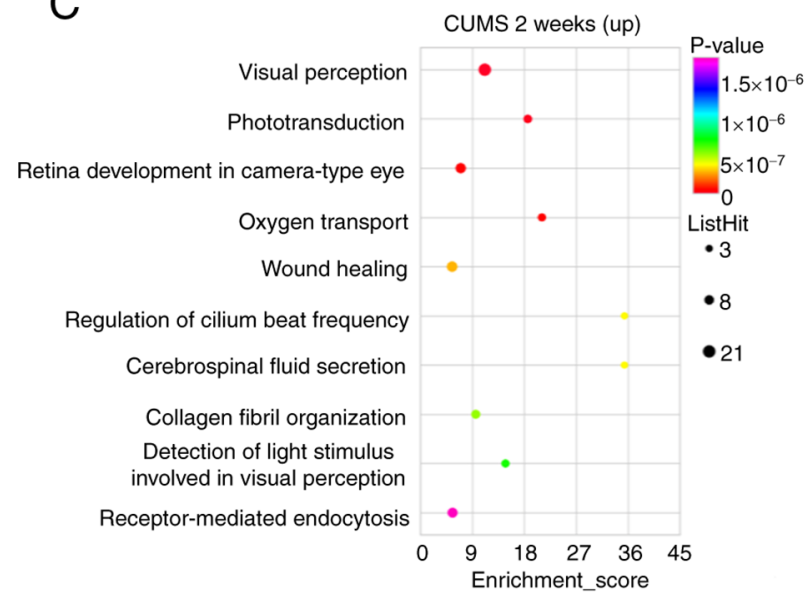

$E$

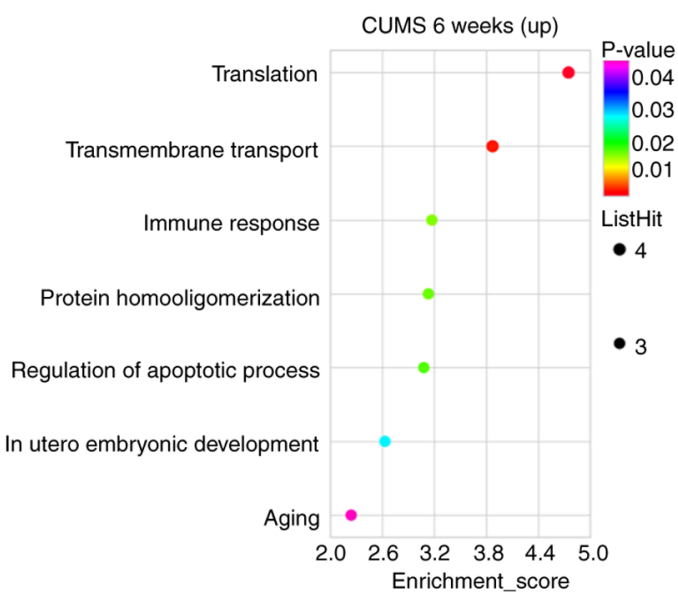

B

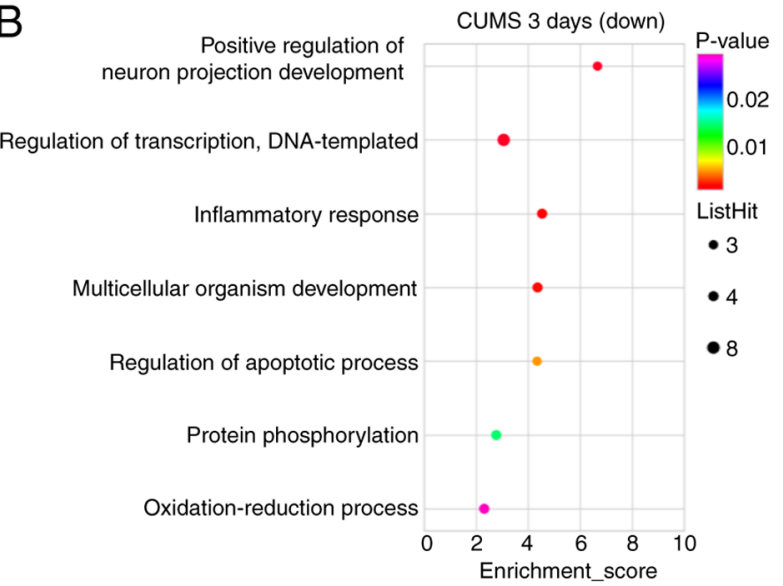

D

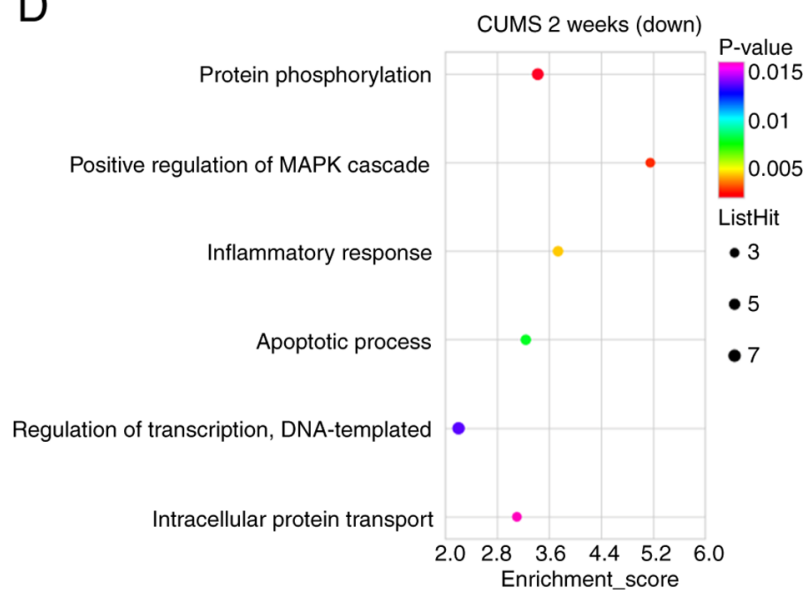

$\mathrm{F}$

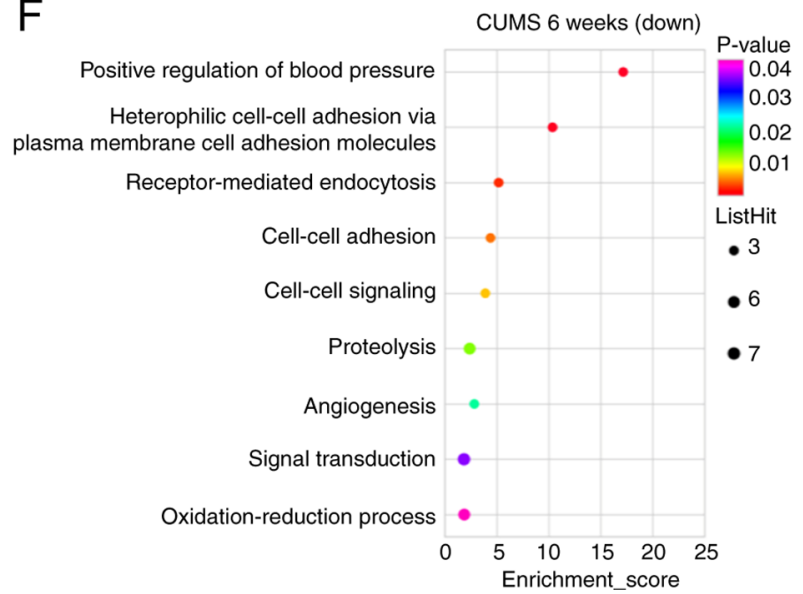

Figure 3. Significant biological processes of the up- and downregulated DEGs of the hippocampus in rats subjected to CUMS for different durations. The top 10 terms with the most significant P-values $(<0.05)$ were selected from enrichment analysis. (A) Up- and (B) downregulated DEGs in CUMS (3 days) rats. (C) Up- and (D) downregulated DEGs in CUMS (2 weeks) rats. (E) Up- and (F) downregulated DEGs in CUMS (6 weeks) rats. CUMS, chronic unpredictable mild stress; DEGs, differentially expressed genes.

and 2 weeks of CUMS. Significant biological processes of upregulated DEGs in CUMS (6 weeks) rats involved 'translation', 'transmembrane transport', 'immune response', 'protein homooligomerization', 'regulation of apoptotic process' and 'aging' (Fig. 3E), while those of the downregulated DEGs in this group included 'positive regulation of blood pressure', 'receptor-mediated endocytosis', 'cell-cell adhesion', 'cell-cell signaling', 'proteolysis', 'angiogenesis', 'signal transduction' and 'oxidation-reduction process' (Fig. 3F). Significant cellular components and molecular functions of DEGs are shown in the supplementary materials (Figs. S2 and S3). 'Integral component of the plasma membrane' was involved in the cellular component of downregulated DEGs in CUMS (3 days) rats and upregulated DEGs in CUMS (6 weeks) rats. 'Protein binding', 'identical protein binding', 'metal ion binding' and 'ATP binding' were involved in the molecular functions of downregulated DEGs in CUMS (3 days) rats and upregulated DEGs in CUMS (6 weeks) rats. Furthermore, 
A

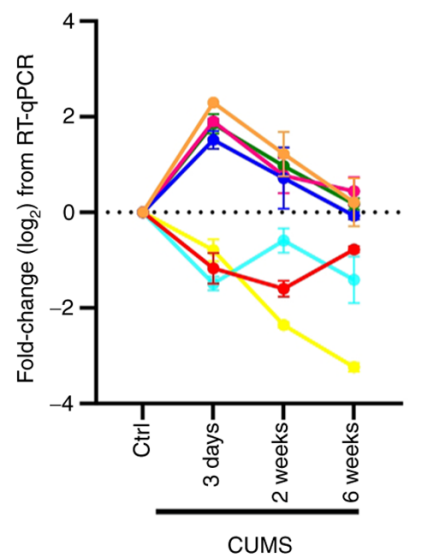

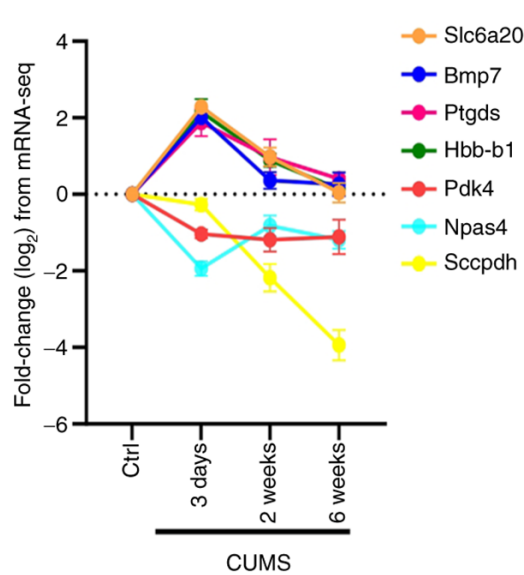

B

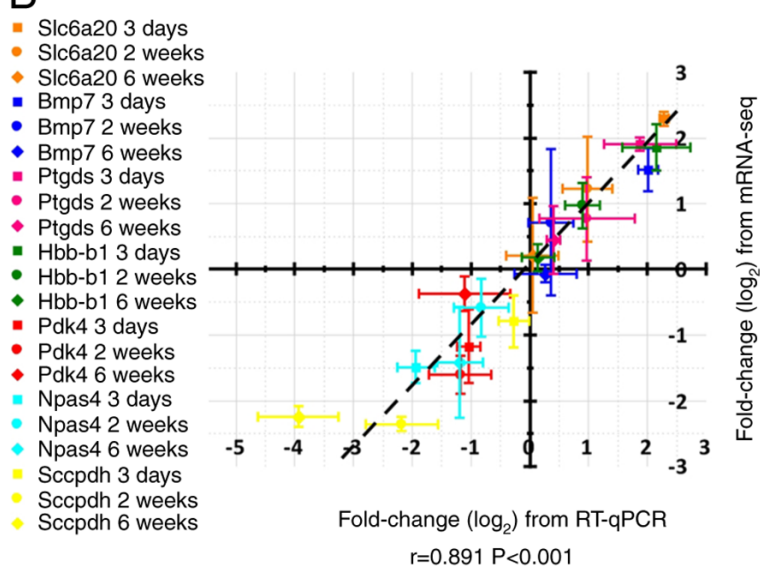

Figure 4. Validation of mRNA-seq data using RT-qPCR. Transcript expression levels from mRNA-seq data were compared with RT-qPCR results. (A) Transcript expression levels detected by mRNA-seq and RT-qPCR. (B) Correlation between expression data from mRNA-seq and RT-qPCR. The data from both methods were consistent. Data are presented as the mean \pm standard deviation $(n=3)$. seq, sequencing; RT-q, reverse transcription-quantitative; CUMS, chronic unpredictable mild stress; Ctrl, control.

significant cellular components of downregulated DEGs in CUMS (6 weeks) rats involved 'synapse', 'dendrite' and 'axon'.

Results of RT-qPCR coincide with the changes in differential gene identified by mRNA-seq. A total of seven differentially expressed transcripts were verified by RT-qPCR. Slc6a20, Bmp7, Ptgds and Hbb-b1 were upregulated during CUMS for 3 days and 2 weeks, and gradually decreased to normal levels at 6 weeks of CUMS. These changes in differential gene detected in mRNA-seq data were confirmed by RT-qPCR. Similarly, Pdk4, Npas4 and Sccpdh were downregulated in the whole CUMS process and their changes identified by RT-qPCR were consistent with mRNA-seq data (Fig. 4A). A significant positive correlation between the expression level data from mRNA-seq and RT-qPCR was identified $(\mathrm{r}=0.891 ; \mathrm{P}<0.001)$ and the slope of the correlation line was 0.915 (Fig. 4B).

\section{Discussion}

In the present study, gene expression profiling of the hippocampus in rats exposed to stress was investigated using mRNA-seq. The GO analysis of DEGs in CUMS for 3 days and 6 weeks shows an inversion of some biological functions. The downregulated genes in CUMS (3 days) rats shared some GO terms with upregulated genes in CUMS (6 weeks) rats, including 'regulation of the apoptotic process', 'integral component of the plasma membrane', 'protein binding', 'identical protein binding', 'metal ion binding' and 'ATP binding'. These results suggested a functional change from adaptation to damage during the early and late stages of chronic stress. Other studies have also reported similar inverted changes in hormones and proteins during stress (22-25). At the onset of stress, glucocorticoids (GCs) exert anti-apoptotic effects targeting protein $\mathrm{Bcl}-2$, while prolonged stress increases free radicals and facilitates apoptosis (22). Moreover, as a key neurotrophic factor, brain-derived neurotrophic factor (BDNF) exhibits a transient increase in the hippocampus following acute stress, whereas chronic stress leads to downregulation of BDNF in the CA3 region of the hippocampus (23-25). The mechanism underlying inverted changes during CUMS is still unknown. Differences between GC receptors [mineralocorticoid receptor (MR) and glucocorticoid receptor (GR)] may account for the inverted changes. GCs at low concentrations in the early stage of stress act via high-affinity MR, which will promote the increase of neuronal excitability. However, higher GC levels in the late stage activate low-affinity GR to exert different effects, such as inhabitation of proliferation and integration (26). Norepinephrine is secreted rapidly in the early stage of stress, while GC levels increase during long-term stress. In the hippocampal dentate gyrus, norepinephrine enhances excitability and synaptic plasticity, but the high levels of corticosterone suppress noradrenergic activity (27). The duration and antagonism of hormones during stress may contribute to the inversion of biological functions. Further studies are needed to investigate the mechanism underlying altered biological processes during chronic stress.

GO analysis of upregulated genes in CUMS (2 weeks) rats revealed vision-associated functional alterations, such as 'visual perception', 'phototransduction', 'retina development in the camera-type eye', 'detection of light stimuli involved in visual perception', 'photoreceptor outer segment', 'photoreceptor inner segment' and 'retinal binding'. This indicated that visual function was significantly altered after 2 weeks of stress, when cognitive impairment had not yet developed. The association between changes in visual function and stress is still unclear. Certain studies have found that stress leads to attentional narrowing and enhanced ability for detection and observation $(28,29)$. However, other studies have found decreased attention and accuracy of visual perceptual processing in stressful states $(30,31)$. The effects of acute stress on visual function are controversial. Chronic stress is hypothesized to be the most common cause of depression. In addition to symptoms such as anxiety, depression is often accompanied by perceptual abnormality, including visual changes; such changes may comprise notable diminished visual acuity or increased sensitivity to light stimuli $(32,33)$. Certain studies have reported visual impairment in patients with depression, including impaired ability to distinguish intensity of light 
in a room and the ability to read $(32,34)$. Golomb et al $(33)$ used a classical visual stimulus, the moving grating test, to test patients with major depressive disorder who had normal visual acuity and found that motion perception was enhanced in patients with depression compared with healthy individuals. The present findings suggested that visual function may be an early indicator of impairment due to stress. Further studies are needed to determine changes in visual perception during stress.

GO analysis of upregulated genes in CUMS (3 days and 2 weeks) rats showed functional changes in 'extracellular matrix' (ECM), 'extracellular space' and 'extracellular region'. GO analysis of downregulated genes in these groups also showed functional changes in 'extracellular space' and 'extracellular region', suggesting an imbalance in synthesis and degradation of extracellular components of hippocampal tissue during the early and middle stages of CUMS. This imbalance might cause excessive deposition of ECM components in the hippocampal tissue (35), such as collagen, metalloproteinase, fibronectin, bone morphogenetic protein 7, nidogen 1 and elastin, which contributes to development of organ fibrosis (36). Collagen is a primary structural component of ECM and serves a key role in the cell cycle stimulated by mitogen (37). However, certain reports have shown that collagen inhibits cell proliferation $(38,39)$. Excessive deposition of fibrous collagen, such as type I collagen, occurs during organ fibrosis, which leads to tissue and organ sclerosis and loss of function. Activation of type I collagen COL1A1 in the hippocampus contributes to the development of hippocampal sclerosis (40). The primary pathological manifestations of hippocampal sclerosis include hippocampal atrophy, neuronal loss, granule cell reorganization, alteration of interneuronal populations and chronic fibrillary gliosis centered on the pyramidal cell layer (41). GO analysis of upregulated DEGs in CUMS (3 days and 2 weeks) groups also indicated a wound healing function. Increased levels of collagen fibers serve an important role in tissue injury healing $(42,43)$. Activation of ECM in the hippocampus during the early and middle stages of CUMS is hypothesized to contribute to self-repair of damaged hippocampal cells but also promotes hippocampal sclerosis, which leads to partial neuronal loss (35). ECM changes are often secondary to inflammatory reactions (36), which indicates that hippocampal inflammation occurs during the early and middle stages of CUMS. Excessive ECM deposition is a damage repair response to hippocampal cell damage and inflammation caused by stress, which contributes to the development of hippocampal sclerosis while promoting self-repair (44).

GO analysis of the upregulated DEGs in CUMS (6 weeks) rats involved changes in 'aging', suggesting that chronic stress shares certain pathways with aging or that long-term chronic stress promotes aging. Aging is often accompanied by pathological signs such as memory loss, Alzheimer's disease, dementia and depression, and chronic stress is one of the contributing factors to these pathological signs $(45,46)$. The results of numerous animal stress experiments and clinical trials show that stress leads to changes in brain structure and function, cognitive decline and accelerated cellular aging $(46,47)$. Sapolsky et al $(48)$ proposed a GC cascade hypothesis of aging, suggesting that stress and $\mathrm{GC}$ accelerate the aging process in rats. The hippocampus is a target and a highly vulnerable area of GC in the brain. Elevated GC levels in the blood of elderly people leads to hippocampal atrophy, the degree of which is positively correlated with degree of GC elevation, resulting in hippocampal dysfunction and deficits in memory and learning and cognitive ability $(49,50)$. In addition, one study found that chronic stress disrupts the normal processing of $\mathrm{A} \beta$ precursor protein, thus leading to the accumulation of $\mathrm{A} \beta$ in the brain (14). The development of physiological aging may interact with certain genetic background or negative environmental conditions (such as chronic stressors and stress in infancy and early childhood) to trigger dysregulation of the hypothalamus-pituitary-adrenal (HPA) axis (51) and changes in the neurotransmitter system and brain structures such as the hippocampus, prefrontal cortex and amygdala (1), making the brain more vulnerable to increased levels of GCs and metabolic changes and less resilient to damage from exposure to stressors $(52,53)$; these alterations lead to formation of an abnormal aging brain with decreased function and neuronal death and stress serves a key role in brain aging.

GO analysis of upregulated DEGs in CUMS (6 weeks) rats showed functional alterations in 'regulation of the apoptotic process' and 'negative regulation of cell proliferation' and downregulated DEGs showed functional alterations in 'receptor-mediated endocytosis', 'angiogenesis', 'synapse', 'dendrite' and 'axon'. This suggested that under chronic stress, neuronal apoptosis increased, multiple functions, such as cell proliferation and endocytosis, were inhibited and expression of genes associated with neuronal structures, such as synapses, axons and dendrites, decreased, resulting in damaging effects, such as impaired neuronal function and synaptic plasticity $(46,49)$. Research has shown that under chronic stress, hippocampal dentate gyrus and CA3 area pyramidal cells atrophy and die (54), mossy fiber-CA3 synaptic transmission (55) dentate gyrus granule cell production decrease, dentate gyrus neurogenesis is inhibited (56) and CA3 area dendritic spine length and number are significantly decreased (57). Chronic stress causes functional and structural damage to hippocampal neurons, which exacerbates neuroendocrine abnormality and leads to mood changes and cognitive impairment (1). Parul et al (58) found that chronic unpredictable stress inhibits hippocampal neurogenesis in adult rats and increases glial cell and neuronal apoptosis in the cerebral cortex and hippocampus, which may be associated with reduced AKT and increased ERK signaling. Dagyte et al (59) concluded that strong activation of the HPA axis during acute stress is not sufficient to decrease hippocampal cell proliferation, but prolonged and repeated exposure to stressful stimuli inhibits hippocampal cell proliferation and leads to neurodevelopmental dysplasia. The present study supported the hypothesis that chronic stress may lead to cumulative changes in the brain that are not observed following acute stress. Such changes may indicate compromised brain plasticity and increased vulnerability to neuropathology (60).

In summary, genome-wide expression data and functional analysis reported here revealed dynamic functional changes during the different stages of CUMS. During the early and middle stages of chronic stress, the hippocampal ECM appears significantly altered and damage repair capacity is enhanced. In the late stage of CUMS, the balance of hippocampal apoptosis and proliferation is disrupted and chronic stress induces 
increased hippocampal apoptosis, aging, and the inhibition of multiple neuronal structures and functions. Further experiments are required to analyzed differential gene expression profiles and provide novel directions for gene therapy to treat chronic stress.

\section{Acknowledgements}

Not applicable.

\section{Funding}

The present study was supported by the National Natural Science Foundation of China (grant nos. 31771290 and 81702454), National Natural Science Foundation of Beijing (grant no. 5222033) and Military Logistics Scientific Research Foundation of China (grant no. BWS17J027).

\section{Availability of data and materials}

The datasets used and/or analyzed during the current study are available from the corresponding author on reasonable request.

\section{Authors' contributions}

LJQ, FX and FL contributed to the conception and design of the study. FL, FX, XW and YZ collected and interpreted the data. FL and FX confirm the authenticity of all the raw data. FL, YW and FX performed statistical analysis and wrote the manuscript. All authors contributed to manuscript revision. All authors have read and approved the manuscript.

\section{Ethics approval and consent to participate}

All experimental protocols were approved by the Institutional Animal Care and Use Committee of the Beijing Institute of Basic Medical Sciences (approval no. IACUC-DWZX-2020670) and were performed in accordance with the NIH Guide for the Care and Use of Laboratory Animals (NIH Publications no. 8023) and the ARRIVE guidelines from NC3Rs. During treatment, efforts were made to minimize the potential pain and distress of animals. Animals were monitored at least four times a week.

\section{Patient consent for publication}

Not applicable.

\section{Competing interests}

The authors declare that they have no competing interests.

\section{References}

1. McEwen BS, Bowles NP, Gray JD, Hill MN, Hunter RG, Karatsoreos IN and Nasca C: Mechanisms of stress in the brain. Nat Neurosci 18: 1353-1363, 2015.

2. Weinberg A and Creed F: Stress and psychiatric disorder in healthcare professionals and hospital staff. Lancet 355: 533-537, 2000.

3. Sinha R: Chronic stress, drug use, and vulnerability to addiction. Ann NY Acad Sci 1141: 105-130, 2008.
4. Rozanski A, Blumenthal JA and Kaplan J: Impact of psychological factors on the pathogenesis of cardiovascular disease and implications for therapy. Circulation 99: 2192-2217, 1999.

5. Bhatia V and Tandon RK: Stress and the gastrointestinal tract. J Gastroenterol Hepatol 20: 332-339, 2005.

6. Reiche EM, Nunes SO and Morimoto HK: Stress, depression, the immune system, and cancer. Lancet Oncol 5: 617-625, 2004.

7. Sapolsky RM: Stress and the brain: Individual variability and the inverted-U. Nat Neurosci 18: 1344-1346, 2015.

8. Kim JJ and Diamond DM: The stressed hippocampus, synaptic plasticity and lost memories. Nat Rev Neurosci 3: 453-462, 2002.

9. Kempermann G and Kronenberg G: Depressed new neurons-adult hippocampal neurogenesis and a cellular plasticity hypothesis of major depression. Biol Psychiatry 54: 499-503, 2003.

10. Chao HM, Ma LY, McEwen BS and Sakai RR: Regulation of glucocorticoid receptor and mineralocorticoid receptor messenger ribonucleic acids by selective agonists in the rat hippocampus. Endocrinology 139: 1810-1814, 1998.

11. Li XH, Chen JX, Yue GX, Liu YY, Zhao X, Guo XL, Liu Q, Jiang YM and Bai MH: Gene expression profile of the hippocampus of rats subjected to chronic immobilization stress. PLoS One 8: e57621, 2013.

12. Ubaldi M, Ricciardelli E, Pasqualini L, Sannino G, Soverchia L, Ruggeri B, Falcinelli S, Renzi A, Ludka C, Ciccocioppo R and Hardiman G: Biomarkers of hippocampal gene expression in a mouse restraint chronic stress model. Pharmacogenomics 16: 471-482, 2015.

13. Antoniuk S, Bijata M, Ponimaskin E and Wlodarczyk J: Chronic unpredictable mild stress for modeling depression in rodents: Meta-analysis of model reliability. Neurosci Biobehav Rev 99: 101-116, 2019.

14. Xie F, Zhao Y, Ma J, Gong JB, Wang SD, Zhang L, Gao XJ and Qian LJ: The involvement of homocysteine in stress-induced $A \beta$ precursor protein misprocessing and related cognitive decline in rats. Cell Stress Chaperones 21: 915-926, 2016.

15. Bolger AM, Lohse $M$ and Usadel B: Trimmomatic: A flexible trimmer for Illumina sequence data. Bioinformatics 30: 2114-2120, 2014.

16. Kim D, Langmead B and Salzberg SL: HISAT: A fast spliced aligner with low memory requirements. Nat Methods 12: 357-360, 2015.

17. Trapnell C, Williams BA, Pertea G, Mortazavi A, Kwan G, van Baren MJ, Salzberg SL, Wold BJ and Pachter L: Transcript assembly and quantification by RNA-Seq reveals unannotated transcripts and isoform switching during cell differentiation. Nat Biotechnol 28: 511-515, 2010.

18. Anders S, Pyl PT and Huber W: HTSeq-a Python framework to work with high-throughput sequencing data. Bioinformatics 31 : 166-169, 2015.

19. Anders S and Huber W: Differential expression analysis for sequence count data. Genome Biol 11: R106, 2010.

20. Ashburner M, Ball CA, Blake JA, Botstein D, Butler H, Cherry JM, Davis AP, Dolinski K, Dwight SS, Eppig JT, et al: Gene ontology: Tool for the unification of biology. The gene ontology consortium. Nat Genet 25: 25-29, 2000.

21. Pfaffl MW: A new mathematical model for relative quantification in real-time RT-PCR. Nucleic Acids Res 29: e45, 2001.

22. Du J, Wang Y, Hunter R, Wei Y, Blumenthal R, Falke C, Khairova R, Zhou R, Yuan P, Machado-Vieira R, et al: Dynamic regulation of mitochondrial function by glucocorticoids. Proc Natl Acad Sci USA 106: 3543-3548, 2009.

23. Daskalakis NP, Cohen H, Cai G, Buxbaum JD and Yehuda R: Expression profiling associates blood and brain glucocorticoid receptor signaling with trauma-related individual differences in both sexes. Proc Natl Acad Sci USA 111: 13529-13534, 2014.

24. Govindarajan A, Rao BS, Nair D, Trinh M, Mawjee N, Tonegawa S and Chattarji S: Transgenic brain-derived neurotrophic factor expression causes both anxiogenic and antidepressant effects. Proc Natl Acad Sci USA 103: 13208-13213, 2006.

25. Lakshminarasimhan $H$ and Chattarji S: Stress leads to contrasting effects on the levels of brain derived neurotrophic factor in the hippocampus and amygdala. PLoS One 7: e30481, 2012.

26. Joëls M and Baram TZ: The neuro-symphony of stress. Nat Rev Neurosci 10: 459-466, 2009.

27. Pu Z, Krugers HJ and Joëls M: Corticosterone time-dependently modulates beta-adrenergic effects on long-term potentiation in the hippocampal dentate gyrus. Learn Mem 14: 359-367, 2007.

28. Clark WC, Yang JC and Janal MN: Altered pain and visual sensitivity in humans: The effects of acute and chronic stress. Ann NY Acad Sci 467: 116-129, 1986. 
29. Tijerina L, Garrott WR, Stoltzfus D and Parmer E: Eye glance behavior of van and passenger car drivers during lane change decision phase. Transp Res Rec 1937: 37-43, 2005.

30. Paul M, Lech RK, Scheil J, Dierolf AM, Suchan B and Wolf OT: Acute stress influences the discrimination of complex scenes and complex faces in young healthy men. Psychoneuroendocrinology 66: 125-129, 2016.

31. Smith KE, Leitzke BT and Pollak SD: Youths' processing of emotion information: Responses to chronic and video-based laboratory stress. Psychoneuroendocrinology 122: 104873, 2020.

32. Norton DJ, McBain RK, Pizzagalli DA, Cronin-Golomb A and Chen Y: Dysregulation of visual motion inhibition in major depression. Psychiatry Res 240: 214-221, 2016.

33. Golomb JD, McDavitt JR, Ruf BM, Chen JI, Saricicek A Maloney KH, Hu J, Chun MM and Bhagwagar Z: Enhanced visual motion perception in major depressive disorder. J Neurosci 29: 9072-9077, 2009.

34. Friberg TR and Borrero G: Diminished perception of ambient light: A symptom of clinical depression? J Affect Disord 61: 113-118, 2000.

35. Anwar MM, Özkan E and Gürsoy-Özdemir Y: The role of extracellular matrix alterations in mediating astrocyte damage and pericyte dysfunction in Alzheimer's disease: A comprehensive review. Eur J Neurosci: Jun 28, 2021 (Epub ahead of print).

36. Rosenberg GA: Extracellular matrix inflammation in vascular cognitive impairment and dementia. Clin Sci (Lond) 131: 425-437, 2017.

37. Hornberger LK, Singhroy S, Cavalle-Garrido T, Tsang W, Keeley F and Rabinovitch M: Synthesis of extracellular matrix and adhesion through beta(1) integrins are critical for fetal ventricular myocyte proliferation. Circ Res 87: 508-515, 2000.

38. Henriet P, Zhong ZD, Brooks PC, Weinberg KI and DeClerck YA Contact with fibrillar collagen inhibits melanoma cell proliferation by up-regulating p27KIP1. Proc Natl Acad Sci USA 97: 10026-10031, 2000.

39. Kelly KK, MacPherson AM, Grewal H, Strnad F, Jones JW, Yu J, Pierzchalski K, Kane MA, Herson PS and Siegenthaler JA: Colla1 ${ }^{+}$perivascular cells in the brain are a source of retinoic acid following stroke. BMC Neurosci 17: 49, 2016

40. Falconer MA, Serafetinides EA and Corsellis JA: Etiology and pathogenesis of temporal lobe epilepsy. Arch Neurol 10: 233-248, 1964.

41. Thom M: Review: Hippocampal sclerosis in epilepsy: A neuropathology review. Neuropathol Appl Neurobiol 40: 520-543, 2014.

42. Rosensteel SM, Wilson RP, White SL and Ehrlich HP: COL1A1 oligodeoxynucleotides decoy: Biochemical and morphologic effects in an acute wound repair model. Exp Mol Pathol 89: 307-313, 2010

43. Shi-Wen X, Leask A and Abraham D: Regulation and function of connective tissue growth factor/CCN2 in tissue repair, scarring and fibrosis. Cytokine Growth Factor Rev 19: 133-144, 2008.

44. Krishnaswamy VR, Benbenishty A, Blinder P and Sagi I: Demystifying the extracellular matrix and its proteolytic remodeling in the brain: Structural and functional insights. Cell Mol Life Sci 76: 3229-3248, 2019.
45. Moreno-FernandezRD,TabbaiS,Castilla-OrtegaE,Perez-MartinM, Estivill-Torrus G, Rodriguez de Fonseca F, Santin LJ and Pedraza C: Stress, depression, resilience and ageing: A role for the LPA-LPA1 pathway. Curr Neuropharmacol 16: 271-283, 2018.

46. de Magalhães JP and Passos JF: Stress, cell senescence and organismal ageing. Mech Ageing Dev 170: 2-9, 2018.

47. Segar TM, Kasckow JW, Welge JA and Herman JP: Heterogeneity of neuroendocrine stress responses in aging rat strains. Physiol Behav 96: 6-11, 2009.

48. Sapolsky RM, Krey LC and McEwen BS: The neuroendocrinology of stress and aging: The glucocorticoid cascade hypothesis. Endocr Rev 7: 284-301, 1986.

49. Raber J: Detrimental effects of chronic hypothalamicpituitary-adrenal axis activation. From obesity to memory deficits. Mol Neurobiol 18: 1-22, 1998.

50. Elgh E, Lindqvist Astot A, Fagerlund M, Eriksson S, Olsson T and Näsman B: Cognitive dysfunction, hippocampal atrophy and glucocorticoid feedback in Alzheimer's disease. Biol Psychiatry 59: 155-161, 2006

51. Buwembo A, Long H and Walker CD: Participation of endocannabinoids in rapid suppression of stress responses by glucocorticoids in neonates. Neuroscience 249: 154-161, 2013.

52. Conrad CD: Chronic stress-induced hippocampal vulnerability: The glucocorticoid vulnerability hypothesis. Rev Neurosci 19: 395-411, 2008

53. Bloss EB, Janssen WG, McEwen BS and Morrison JH: Interactive effects of stress and aging on structural plasticity in the prefrontal cortex. J Neurosci 30: 6726-6731, 2010.

54. Magariños AM, McEwen BS, Flügge G and Fuchs E: Chronic psychosocial stress causes apical dendritic atrophy of hippocampal CA3 pyramidal neurons in subordinate tree shrews. J Neurosci 16: 3534-3540, 1996.

55. Magariños AM, Verdugo JM and McEwen BS: Chronic stress alters synaptic terminal structure in hippocampus. Proc Natl Acad Sci USA 94: 14002-14008, 1997.

56. Gould E and Tanapat P: Stress and hippocampal neurogenesis. Biol Psychiatry 46: 1472-1479, 1999.

57. McEwen BS: Stress and hippocampal plasticity. Annu Rev Neurosci 22: 105-122, 1999.

58. Parul, Mishra A, Singh S, Singh S, Tiwari V, Chaturvedi S, Wahajuddin M, Palit G and Shukla S: Chronic unpredictable stress negatively regulates hippocampal neurogenesis and promote anxious depression-like behavior via upregulating apoptosis and inflammatory signals in adult rats. Brain Res Bull 172: 164-179, 2021

59. Dagyte G, Van der Zee EA, Postema F, Luiten PG, Den Boer JA, Trentani A and Meerlo P: Chronic but not acute foot-shock stress leads to temporary suppression of cell proliferation in rat hippocampus. Neuroscience 162: 904-913, 2009.

60. McEwen BS and Morrison JH: The brain on stress: Vulnerability and plasticity of the prefrontal cortex over the life course. Neuron 79: 16-29, 2013

This work is licensed under a Creative Commons Attribution-NonCommercial-NoDerivatives 4.0 International (CC BY-NC-ND 4.0) License. 